\title{
Update on polycystic kidney disease (hereditary): genetic diagnosis and counseling
}

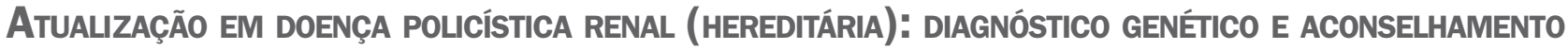 \\ Wanderley M. Bernardo, Martin R. Whittle e Ricardo Simões
}

http://dx.doi.org/10.1590/1806-9282.60.03.003

1. In prenatal and neonatal context, is ultrasonography sufficient to confirm the clinical diagnosis of autosomal recessive polycystic kidney disease (ARPKD)?

a. Ultrasound examination is not the $1^{\text {st }}$ investigation to be applied to fetuses and neonates with suspected disease.

b. Yes, without the need for other tests.

c. Renal ultrasound abnormalities are detectable from 32 weeks of gestation.

d. Renal ultrasound abnormalities are detectable from the 13th week of pregnancy when the diagnosis was previously established in an affected sibling.

2. In the context of an adult, if the result of the ultrasound examination is inconclusive, does the molecular test allow reaching a definitive conclusion?

a. Molecular tests may be indirect, such as PKHD1 gene sequencing, or indirect, using linkage analysis.

b. Molecular tests can be direct, such as linkage analysis.

c. The type and position of mutations in the PKHD1 gene provide information about the prognosis of the disease.

d. Direct molecular genetic testing can detect all mutations causing ARPKD.

3. Does ultrasound examination allow confirming the clinical diagnosis of autosomal dominant polycystic kidney disease (ADPKD)?

a. In patients aged 15 to 29 years with 3 or more unilateral or bilateral cysts, the sensitivity is $69.5 \%$ and specificity is $100 \%$.

b. In patients aged 40 to 59 years with 2 or more unilateral or bilateral cysts, the sensitivity is $70 \%$ and specificity is $78 \%$.

c. Patients aged over 60 years with 4 or more cysts in each kidney, sensitivity is $1 \%$ and specificity is $1 \%$.

d. Investigation using ultrasound is not recommended as a first choice.
4. What are the advantages and disadvantages of indirect versus direct approaches in molecular testing for ADPKD?

a. Genetic linkage analysis (using polymorphic markers within and / or near the genes that define haplotypes) complements the indirect tests.

b. Haplotype analysis is quick, simple and inexpensive.

c. Indirect studies can be made in a single patient, but are costly, time consuming and expensive and do not always provide definitive information.

d. Gene sequencing is the most direct.

5. What is the role of molecular testing for genetic counseling of a couple or family that carries ADPKD?

a. Molecular tests are the only investigation that can provide predictive information about ADPKD in individuals before clinical signs and symptoms develop.

b. The type of mutations in the genes provides Information about the disease's diagnosis.

c. Gene rearrangements comprise around $40 \%$ of the molecular lesions.

d. In all families the disease develops similarly among affected siblings.

ANSWERS TO CLINICAL SCENARIO: UPDATE ON VACCINATION FOR THE PREVENTION OF INFECTIOUS RESPIRATORY DISEASE IN DDULTS [PUBLISHED IN RAMB 2014; 60(2)]

1. Is there benefit in vaccine combination for the prevention of infectious respiratory diseases in adults?

Both the anti-influenza and pneumococcal vaccines reduce hospitalizations. (alternative $\mathrm{B}$ )

2. Are there any differences between pneumococcal polysaccharide vaccines (VPPS-23) and conjugate vaccines?

The pneumococcal vaccine is not recommended for pregnant women. (alternative C) 
3. Regardin the use of BCG vaccines, it is correct to say that:

The BCG vaccine is recommended for newborns through their first month of life. (alternative A)

4. Are there benefits in using anti-pertussis vaccines? Adults who live or work with infants or children under 1 year old should receive a single booster. (alternative $\mathrm{D}$ )
5. What are the indications for pneumococcal vaccine?

Anyone between 2 and 64 years old who has a chronic disease. (alternative $\mathrm{B}$ ) 